\title{
Feasibility of a Two-Metal, Four-Purine Nucleobase Quartet Motif
}

Michael Roitzsch and Bernhard Lippert*

Fachbereich Chemie, Universität Dortmund, 44221 Dortmund, Germany

\section{Supporting Information: $p D$ dependent ${ }^{1} H$ NMR spectroscopy displaying the} acid-base equilibrium of the adenine ligand.

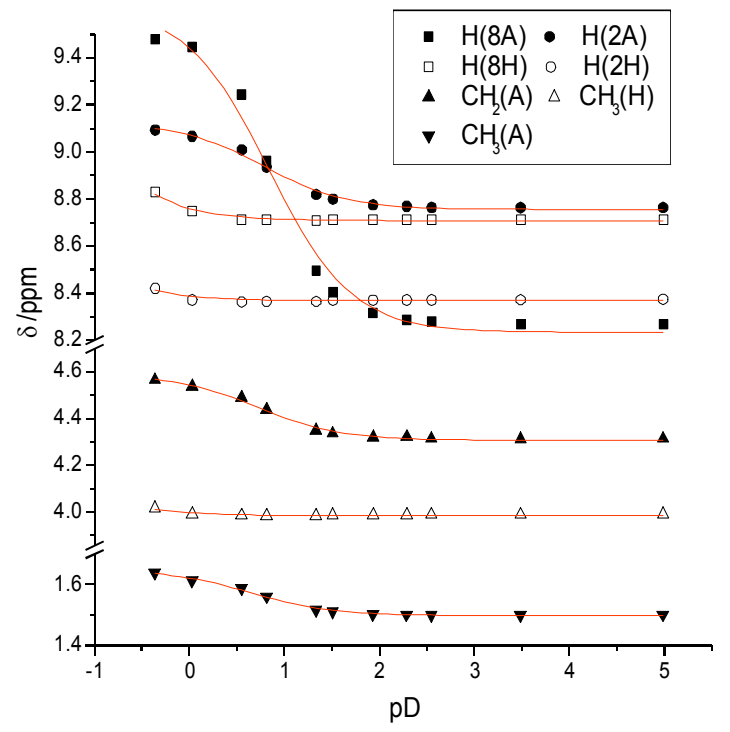

For least-squares fitting the following equation was used ${ }^{1}$

$\delta_{\mathrm{obs}}=\frac{\delta_{\mathrm{B}}+\delta_{\mathrm{HB}^{+}} \cdot 10^{\left(\mathrm{p} K_{\mathrm{HB}^{+}}-\mathrm{pD}\right)}}{1+10^{\left(\mathrm{pK}_{\mathrm{HB}^{+}} \mathrm{pD}\right)}}$

The $\mathrm{p} K_{\mathrm{a}}$ values obtained by this procedure are valid for heavy water. Transformation for $\mathrm{H}_{2} \mathrm{O}$ was calculated by the following equation $^{2}$

$\mathrm{p} K^{\mathrm{H}_{2} \mathrm{O}}=\frac{\mathrm{p} K^{\mathrm{D}_{2} \mathrm{O}}-0.45}{1.015}$

Figure 1: Plot of the chemical shifts of trans$\left[\left(\mathrm{NH}_{3}\right)_{2} \mathrm{Pt}(9-\mathrm{EtA}-\mathrm{N1})(9-\mathrm{MeHxH}-\mathrm{N} 7)\right]^{2+}$ vs. $\mathrm{pD}$ value.

Tabular 1: Parameters of the non-linear least-squares fits

\begin{tabular}{lcccc}
\hline Parameter & $\mathrm{pK}^{\mathrm{D} 2 \mathrm{O}}$ & $\mathrm{pK}^{\mathrm{H} 2 \mathrm{O}}$ & $\delta\left(\mathrm{HB}^{+}\right)$ & $\delta(\mathrm{B})$ \\
\hline $\mathrm{H}(8 \mathrm{~A})$ & $0.84(7)$ & $0.38(7)$ & $9.62(6)$ & $8.23(3)$ \\
$\mathrm{H}(2 \mathrm{~A})$ & $0.78(6)$ & $0.33(6)$ & $9.12(1)$ & $8.756(6)$ \\
$\mathrm{CH}_{2}(\mathrm{~A})$ & $0.72(6)$ & $0.27(6)$ & $4.59(1)$ & $4.308(4)$ \\
$\mathrm{CH}_{3}(\mathrm{~A})$ & $0.64(6)$ & $0.19(6)$ & $1.65(1)$ & $1.497(2)$ \\
$\mathrm{H}(8 \mathrm{H})$ & & & 8.709 \\
$\mathrm{H}(2 \mathrm{H})$ & & & 8.367 \\
$\mathrm{CH}_{3}(\mathrm{H})$ & & & 3.985 \\
\hline
\end{tabular}

\section{References}

${ }^{1}$ Tribolet, R.; Sigel, H. Eur. J. Biochem. 1987, 163, 353-363.

${ }^{2}$ Martin, R. B. Science 1963, 139, 1198-1203. 